\title{
Frequency-dependent transport through a quantum dot in the Kondo regime
}

\author{
M. Sindel, ${ }^{1}$ W. Hofstetter, ${ }^{2}$ J. von Delft, ${ }^{1}$ and M. Kindermann ${ }^{2}$ \\ ${ }^{1}$ Physics Department, Arnold Sommerfeld Center for Theoretical Physics, \\ and Center for NanoScience, Ludwig-Maximilians-Universität München, 80333 München, Germany \\ ${ }^{2}$ Department of Physics, Massachusetts Institute of Technology, Cambridge MA 02139, USA
}

(Dated: October 9, 2018)

\begin{abstract}
We study the AC conductance and equilibrium current fluctuations of a Coulomb blockaded quantum dot in the Kondo regime. To this end we have developed an extension of the numerical renormalization group suitable for the nonperturbative calculation of finite-frequency transport properties. We demonstrate that AC transport gives access to the many-body resonance in the equilibrium spectral density. It provides a new route to measuring this key signature of Kondo physics which so far has defied direct experimental observation.
\end{abstract}

In the past years semiconductor quantum dots have gained considerable attention as tunable magnetic impurities 1]. Due to their small size, electronic transport through these structures is strongly influenced by the Coulomb blockade [2]. Quantum dots with an odd number of electrons display a well-known many-body phenomenon, the Kondo effect [3, 4], as predicted in [5]. In these systems, a single unpaired spin is screened at low temperatures, giving rise to an enhanced DC (zerofrequency) conductance at low bias voltage.

Theoretical studies have so far mostly focused on DC transport [5]. From the differential conductance $d I / d V$ at finite bias voltage $V$ one can obtain information about the Kondo resonance in the spectral density. Such measurements on a typical, symmetrically coupled quantum dot, however, access only the spectral function under non-equilibrium conditions. It is known that in this case a finite bias splits and suppresses the Kondo peak []. It has also been demonstrated experimentally that a sufficiently strong AC modulation of the gate or bias voltage likewise suppresses the Kondo peak 7, 8]. Thus, it is by no means straightforward to measure the equilibrium spectral function of a Kondo quantum dot. Previous proposals to do so require either very asymmetric couplings to the leads or quantum dots in a 3-terminal geometry 9, 10].

In this Letter we point out that the Kondo resonance in the equilibrium spectral density of a generic quantum dot can be extracted from AC transport measurements. We derive a direct relation between the spectral function and the linear AC conductance through a quantum dot [Eq. (52) below]. Alternatively, the Kondo peak can be measured by detecting equilibrium fluctuations in the current through the quantum dot at frequencies of the order of the Kondo temperature $T_{K}$ [Eq. [8] below]. Such measurements have come into experimental reach with recent advances in the measurement of high-frequency current fluctuations [11]. Conductance measurements in the relevant frequency regime are challenging, mainly due to parasitic currents through hardly avoidable capacitances. One should, however, be able to extract the signal due to the current through the quantum dot from this back-

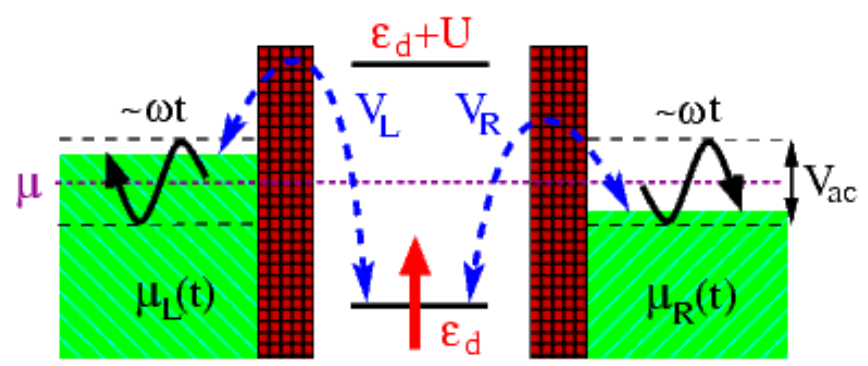

FIG. 1: Single-level Anderson model with time dependent chemical potential $\mu_{\alpha}(t)=\mu \pm\left(e V_{\text {ac }} / 2\right) \cos \omega t$ in lead $\alpha \in$ $\{L, R\}$.

ground by an adiabatic change (much slower than the inverse Kondo temperature 12]) of gate voltage that drives the dot periodically into and out of the Kondo regime.

We apply the nonperturbative numerical renormalization group (NRG) in combination with the Kubo formalism [13, 14] to predict the frequency-dependence of the $\mathrm{AC}$ conductance and the equilibrium noise. Our results are numerically essentially exact, in contrast to previous perturbative calculations [15], and generalize those of 14] to finite frequency. This allows us to access the experimentally most relevant regime of frequencies of the order of the Kondo temperature for a device that exhibits a strong Kondo effect.

The exact correspondence between spectral function and $\mathrm{AC}$ conductance holds only when charge fluctuations on the quantum dot are negligible. Using our rigorous NRG results, we verify that this condition is well satisfied for frequencies below the charging energy of the dot.

The setup that we study is shown in Fig. 1] At low energies, a Coulomb-blockaded quantum dot is well described by the single-level Anderson model

$$
\begin{aligned}
H= & \sum_{\sigma} \epsilon_{d \sigma} d_{\sigma}^{\dagger} d_{\sigma}+U n_{d \uparrow} n_{d \downarrow}+\sum_{k \alpha \sigma} V_{\alpha}\left(d_{\sigma}^{\dagger} c_{k \alpha \sigma}+\text { h.c. }\right) \\
& +\sum_{\substack{k \sigma \\
\alpha \in\{L, R\}}}\left[\epsilon_{k}-\mu_{\alpha}(t)\right] c_{k \alpha \sigma}^{\dagger} c_{k \alpha \sigma}
\end{aligned}
$$

where the chemical potentials $\mu_{L(R)}(t)=\mu \pm$ 
$\left(e V_{\mathrm{ac}} / 2\right) \cos \omega t$ for the left (right) lead include a timedependent bias $V_{\mathrm{ac}}$. We assume that the AC perturbation does not couple to electrons in the local level $d_{\sigma}\left(n_{d \sigma}=d_{\sigma}^{\dagger} d_{\sigma}\right) . \quad V_{\alpha}$ couples the level $d$ to electron states $c_{k \alpha \sigma}$ with momentum $k$ and spin $\sigma= \pm 1$ in lead $\alpha \in\{L, R\}$. In the following, $D$ denotes the conduction electron bandwidth. $\epsilon_{d \sigma} \equiv \epsilon_{d}+\sigma B^{*} / 2, B^{*}=g \mu_{B} B$, is the energy of the local level, including the Zeeman shift in presence of a magnetic field $B . U$ is the Coulomb repulsion of electrons on the dot.

Following [16] we relate the current $I$ through the quantum dot to the local Green function of the level $d$. For frequencies $\hbar \omega \ll \Delta_{c}$ much smaller than the charge excitation energy $\Delta_{c}=\min \left\{\left|\epsilon_{d}\right|,\left|U+\epsilon_{d}\right|\right\}$, charge fluctuations on the quantum dot can be neglected and the currents flowing through the right and the left lead are equal to a good approximation: $I_{L}=-I_{R}$. It is then advantageous to write the total current as $I=\left(I_{L}-\lambda I_{R}\right) /(1+\lambda)$, where $\lambda=\Gamma_{L} / \Gamma_{R}, \Gamma_{\alpha}=\pi \nu V_{\alpha}^{2}$ and $\nu$ is the conduction electron density of states. Expressing the currents $I_{L}$ and $I_{R}$ by the Green function of $d$ (see Eq. (15) in [16]) and Fourier transforming from time $t$ to frequency $\bar{\omega}$, we find for energy-independent couplings $\Gamma_{\alpha}$ the current

$$
\begin{aligned}
I(\bar{\omega}) & =-\frac{2 e^{2} V_{\mathrm{ac}}}{\hbar^{2} \omega} \frac{\lambda}{1+\lambda} \Gamma_{R} \int_{-\infty}^{\infty} d t e^{i \bar{\omega} t} \int_{-\infty}^{\infty} d t_{1} \int \frac{d \epsilon}{2 \pi} \\
& \times \operatorname{Re}\left[e^{i \epsilon\left(t-t_{1}\right)}\left(\sin \omega t-\sin \omega t_{1}\right) f(\epsilon) \mathcal{G}^{r}\left(t, t_{1}\right)\right]
\end{aligned}
$$

in linear response to $V_{\mathrm{ac}}$. Here $f(\epsilon)$ is the equilibrium Fermi function of the leads and $\mathcal{G}^{r}\left(t_{1}, t_{2}\right)=-i \theta\left(t_{1}-t_{2}\right)\left\langle\left\{d\left(t_{1}\right), d^{\dagger}\left(t_{2}\right)\right\}\right\rangle$ the retarded Green function of $d$ evaluated in equilibrium $\left(V_{\mathrm{ac}}=0\right)$. From Eq. (2) we obtain the real $\left(G^{\prime}\right)$ and imaginary $\left(G^{\prime \prime}\right)$ parts of the linear conductance,

$$
\begin{gathered}
G^{\prime}(\omega)=\frac{e^{2}}{2 \hbar^{2} \omega} \frac{\Gamma_{L} \Gamma_{R}}{\Gamma} \int d \epsilon f(\epsilon)[A(\epsilon+\omega)-A(\epsilon-\omega)], \\
\begin{array}{r}
G^{\prime \prime}(\omega)=\frac{e^{2}}{\hbar^{2} \omega} \frac{\Gamma_{L} \Gamma_{R}}{\Gamma} \int \frac{d \epsilon}{2 \pi} f(\epsilon)\left[-2 \operatorname{Re} \mathcal{G}^{r}(\epsilon)\right. \\
\left.+\operatorname{Re} \mathcal{G}^{r}(\epsilon+\omega)+\operatorname{Re} \mathcal{G}^{r}(\epsilon-\omega)\right],
\end{array}
\end{gathered}
$$

where $\Gamma=\Gamma_{L}+\Gamma_{R}$ and $A(\omega)=-\frac{1}{\pi} \operatorname{Im} \mathcal{G}^{r}(\omega)$ is the spectral function. At zero temperature $k T \ll \hbar|\omega| \ll \Delta_{c}$ Eq. (3) is readily solved for the symmetrized spectral function $A_{s}(\epsilon)=[A(\epsilon)+A(-\epsilon)] / 2$,

$$
A_{s}(\omega)=\frac{\hbar^{2}}{e^{2}} \frac{\Gamma}{\Gamma_{L} \Gamma_{R}} \frac{\partial}{\partial \omega}\left[\omega G^{\prime}(\omega)\right] .
$$

In the Kondo regime $\left(\left\langle n_{d}\right\rangle \simeq 1\right)$, the Kondo resonance is centered almost exactly around 0, thus the Kondo peaks in $A_{s}(\omega)$ and $A(\omega)$ are essentially indistinguishable. Hence, Eq. (5) allows us to extract the Kondo peak in the equilibrium spectral function from a measurement of the AC conductance.
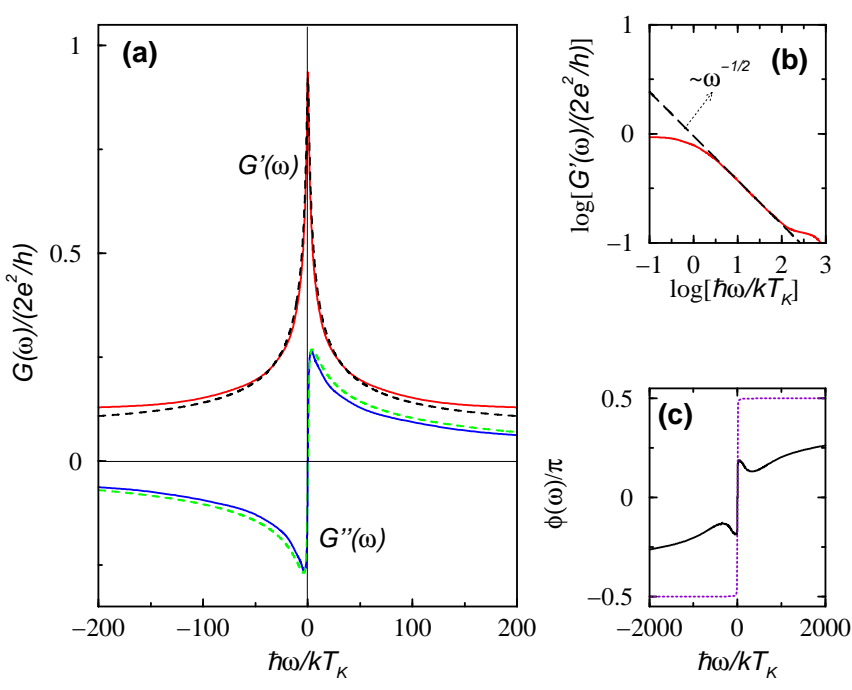

FIG. 2: (a) Real and imaginary parts of the AC conductance obtained via the Kubo approach (solid lines) and from the conductance formulas Eqs. (3) and (4) (dashed lines). Note the excellent agreement for frequencies $\hbar \omega \lesssim 100 k T_{K}$ $\left(\Delta_{c} \approx 600 k T_{K}\right)$. (b) Doniach-Sunjić tails in $A(\omega)$ are responsible for the power-law behavior (dashed line) in the conductance (solid line) which is well described by $G^{\prime} \propto \omega^{-1 / 2}$ for $20 k T_{K}<\omega<200 k T_{K}$ [17]. (c) Comparison of the frequency-dependent conductance phase $\phi(\omega)$ in the Kondo regime (solid line) (parameters $U=0.12 D, U / \Gamma=6, \epsilon_{d}=$ $-U / 2, T=0)$ with that for a resonant level $\left(\epsilon_{d}=0\right)$ of width $k T_{K}$ (dotted line).

The frequency-dependent equilibrium current fluctuations (Johnson-Nyquist noise)

$$
C(\omega)=\int_{-\infty}^{\infty} d t e^{i \omega t}\left[\langle I(0) I(t)\rangle-\langle I\rangle^{2}\right]
$$

are related to the linear conductance by the fluctuation dissipation theorem (FDT)

$$
C(\omega)=\frac{2 \hbar \omega}{\exp (\hbar \omega / k T)-1} G^{\prime}(\omega) .
$$

Consequently, the spectral function can alternatively be inferred from a measurement of $C(\omega)$. At zero temperature, $k T \ll \hbar|\omega| \ll \Delta_{c}$, we arrive at

$$
A_{s}(|\omega|)=-\frac{\hbar}{2 e^{2}} \frac{\Gamma}{\Gamma_{L} \Gamma_{R}} \frac{\partial}{\partial|\omega|} C(-|\omega|) .
$$

Note that at zero temperature $C(\omega)$ is non-vanishing only for $\omega<0$, that is fluctuations have to be measured by probing absorption by the quantum dot. In 11] the measurement of current fluctuations at frequencies up to $\omega \simeq 100 \mathrm{GHz}$ has been reported. This frequency scale is of the same order of magnitude as the Kondo temperature in typical Kondo quantum dots [1]. It makes noise measurements a promising candidate for 
experimental studies of the Kondo peak in the spectral function of a quantum dot.

We turn now to a discussion of the frequency dependence of $\mathrm{AC}$ conductance and equilibrium noise in a Kondo quantum dot with typical experimental parameters. We apply the numerical renormalization group (NRG), first used by Wilson to solve the Kondo problem [13. This method is nonperturbative and does not suffer from low-energy divergences common to scaling approaches. In particular, it provides accurate results in the most interesting crossover regime $\hbar \omega \lesssim k T_{K}$. Within NRG we apply two independent approaches: Using the Kubo formula, we are able to calculate the conductance in the Anderson model Eq. (11) numerically exactly. On the other hand, using Eqs. (3) and (7), we obtain conductance and noise from the single-particle Green function of the $d$ level, that we determine by NRG as well. By comparing the two approaches we shall demonstrate the validity of the approximation underlying Eqs. (3) and (4) for frequencies of the order of the Kondo scale.

We apply the Kubo formalism following Izumida et al. [14]. We define an electric current from lead $L$ to $R$ as $\hat{I}=\frac{e}{2}\left[\left\langle\dot{N}_{R}\right\rangle-\left\langle\dot{N}_{L}\right\rangle\right]$, where $N_{\alpha}=\sum_{k \sigma} c_{k \alpha \sigma}^{\dagger} c_{k \alpha \sigma}$ is the total number of electrons in lead $\alpha$ and $\dot{N}_{\alpha}=$ $\frac{i}{\hbar}\left[H, N_{\alpha}\right]$. Introducing a linear response tensor $\sigma$ by $\left\langle\dot{N}_{\alpha}\right\rangle=\sigma_{\alpha \beta}(\omega) \mu_{\beta}^{\prime}$, where $\mu_{L(R)}^{\prime}= \pm\left(e V_{\mathrm{ac}} / 2\right) \cos \omega t$ is the time-dependent bias applied to the left (right) lead, the total linear $\mathrm{AC}$ conductance takes the form

$$
G(\omega)=\frac{e^{2}}{4}\left[\sigma_{L L}(\omega)+\sigma_{R R}(\omega)-\sigma_{L R}(\omega)-\sigma_{R L}(\omega)\right] .
$$

The complex response tensor $\sigma$ can be written as $\sigma_{\alpha \beta}(\omega)=\frac{1}{i \omega}\left[K_{\alpha \beta}(\omega)-K_{\alpha \beta}(0)\right]$, where

$$
K_{\alpha \beta}(\omega)=-\frac{i}{\hbar} \int_{0}^{\infty} d t e^{-\delta t+i \omega t}\left\langle\left[\dot{N}_{\beta}(0), \dot{N}_{\alpha}(t)\right]\right\rangle
$$

with $\delta \rightarrow 0^{+}$. $\langle\ldots\rangle$ in Eq. (10) refers to the equilibrium expectation value with respect to the Hamiltonian $H$ with $V_{\text {ac }}=0$. We evaluate this expression using NRG 13. From the matrix elements of the current operator $\langle n|\hat{I}| m\rangle$ the imaginary part $K_{\alpha \beta}^{\prime \prime}$ is obtained directly while the real part $K_{\alpha \beta}^{\prime}$ can be calculated via a KramersKronig transformation.

In Fig. 2] we compare the conductance obtained from Eq. (3) with the result of the calculation in the Kubo formalism for $\epsilon_{d}=-U / 2$ and $B=0$. We find excellent agreement for frequencies below the charge gap $\Delta_{c}$. Moreover the large frequency $\left(\hbar \omega \gg k T_{K}\right)$ asymptotes of the conductance reveal a decay of $G^{\prime}(\omega) \sim \omega^{-1 / 2}$ [Fig. 2(b)]. This is expected as a consequence of the Doniach-Sunjić tails of the spectral function [17] together with Eq. (51). The deviation of $G^{\prime}(\omega)$ from the unitary limit (by $\sim 6 \%$ ) is due to systematic numerical errors accumulating in the NRG procedure. Fig. 2(c) shows

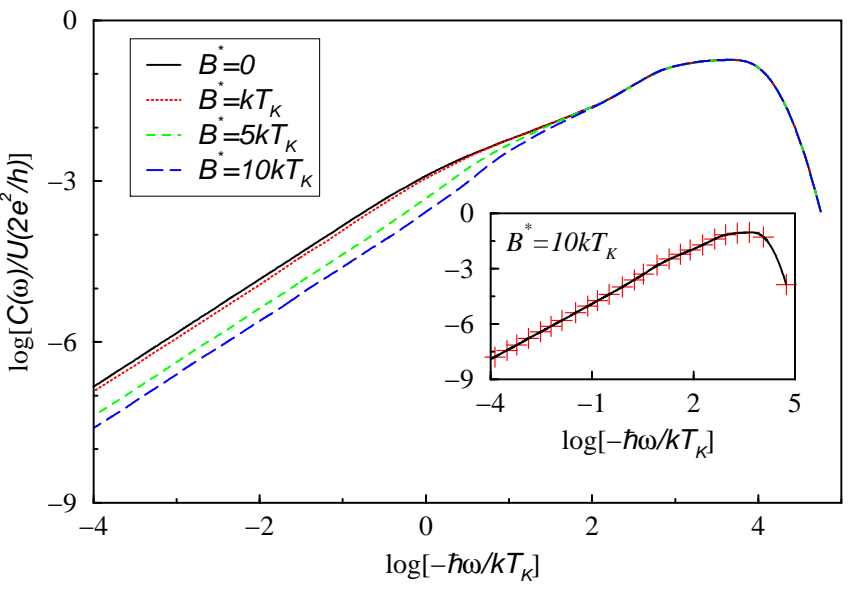

FIG. 3: The equilibrium current fluctuations $C(\omega)$ depend linearly on $\omega$ for $\hbar|\omega| \leq k T_{K}$ [20], as shown for various magnetic fields $B$. The inset shows that the fluctuations are spinindependent even in presence of a finite magnetic field $B^{*}$ $\left(B^{*}=10 k T_{K}\right.$, (red) crosses: $\sigma=\uparrow$, (black) solid line: $\left.\sigma=\downarrow\right)$. Parameters: $U=0.12 D, U / \Gamma=6, \epsilon_{d}=-U / 2, T=0$.

the frequency-dependent phase $\phi(\omega)$ of the linear conductance, $G(\omega)=|G(\omega)| e^{i \phi(\omega)}$.

The frequency-dependence of the equilibrium noise obtained by a direct numerical evaluation of Eq. (6) within NRG is plotted in Fig. 3. As one expects, the fluctuations reach a maximum for frequencies $\hbar \omega \sim U$. The linear behavior $C(\omega) \sim \omega$ at small $\omega$ is a manifestation of the Fermi-liquid nature of a screened Kondo impurity. Fig. 3 also shows how a finite magnetic field $B$ suppresses lowfrequency equilibrium fluctuations. The inset demonstrates that at low frequencies the noise for spin up and spin down electrons is identical, even in the presence of a magnetic field. This is because an impurity with large charging energy $\Delta_{c} \gg \Gamma$ is half-filled: $\left\langle n_{d \uparrow}\right\rangle+\left\langle n_{d \downarrow}\right\rangle=1$. As a consequence of the Friedel sum rule [18], the spectral densities at the Fermi energy $\epsilon_{F}$ for spin-up and spindown electrons $A_{\uparrow / \downarrow}\left(\epsilon_{F}\right) \propto \sin ^{2}\left(\pi\left\langle n_{d \uparrow / \downarrow}\right\rangle\right)$ are then equal.

We now use Eq. (8) to extract the spectral function $A_{s}$ from the numerical current noise data of Fig. 3 where charge fluctuations on the dot have been taken into account. The results are shown in Fig. 4 They nicely demonstrate the splitting and the suppression of the Kondo peak upon increasing the external field $B$. A comparison with the spectral function directly calculated by NRG confirms that Eq. (8) does indeed work very well for frequencies $\hbar \omega \ll \Delta_{c}$. In particular, the Kondo peak in the symmetrized spectral function $A_{s}(\omega)$ obtained by our method is essentially indistinguishable from that in the spectral function $A(\omega)$ directly calculated by NRG. Due to intrinsic broadening in the NRG which affects both $A(\omega)$ and $C(\omega)$, Eq. (8) becomes less accurate for finite $B$.

Having demonstrated that neglecting charge fluctuations in deriving Eqs. (5) and (8) is very well justified, 


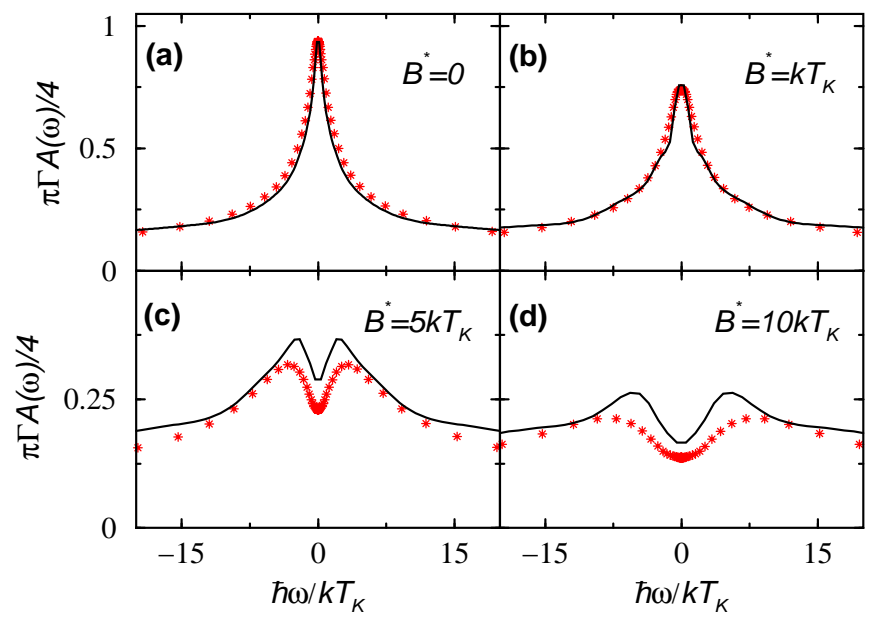

FIG. 4: Spectral function $A(\omega) \equiv A_{s}(\omega)$ as extracted from the current noise $C(\omega)$ via Eq. (8) (lines) compared to $A(\omega)$ obtained directly by NRG (stars) for different values of the magnetic field $B$. Eq. (8) captures the Kondo peak in $A(\omega)$ very well, including suppression and splitting in presence of a magnetic field $B$.

we comment on another limitation of our approach. Calculating the linear response of the quantum dot, we have assumed the bias voltage in a conductance measurement to be small. While this in itself is not a problem, one might worry, however, that due to the finite frequency of $V_{a c}$ extra decoherence processes would make the limit of linear response very restrictive. The decoherence rate $\tau$ of the dot's spin due to the oscillating bias voltage for $\hbar \omega \gg k T_{K}$ can be estimated [19] as

$$
\frac{\hbar \tau^{-1}}{k T_{K}} \sim\left(\frac{e V_{\mathrm{ac}}}{k T_{K}}\right)^{2} \frac{k T_{K}}{\hbar \omega} \frac{1}{\left[\ln \left(\hbar \omega / k T_{K}\right)\right]^{2}} .
$$

For the Kondo physics not to be disrupted by $V_{\mathrm{ac}}$, we need $\hbar \tau^{-1} / k T_{K} \ll 1$. Eq. (11) shows that this condition can be easily fulfilled by the usual requirement $e V_{\text {ac }} \ll k T_{K}$. We expect that this statement remains true for frequencies of the order of $T_{K}$. We conclude that transport is described accurately by our linear response conductance for voltages that are much smaller than the Kondo temperature.

In conclusion, we have studied AC transport through a quantum dot in the Kondo regime, using the numerical renormalization group technique in combination with the Kubo formalism. We have expressed linear conductance and equilibrium current fluctuations in terms of the single-particle Green function. This relation becomes exact at low frequencies, when charge fluctuations on the dot can be neglected. It has been shown to work very well for frequencies of the order of the Kondo scale. This opens up the exciting possibility of measuring the equilibrium Kondo resonance directly in a transport measurement.
Acknowledgements: We would like to thank S. Amasha, L. Borda, M. Kastner, and A. Kogan for valuable discussions, and L. Glazman for comments on the manuscript. Financial support from SFB 631 of the DFG and CeNS is gratefully acknowledged. W.H. has been supported by a Pappalardo grant; M.K. by the Cambridge-MIT Institute Ltd.

[1] L. Kouwenhoven and L. Glazman, Physics World 14, 33 (2001).

[2] L.P. Kouwenhoven et al. in Mesoscopic Electron Transport, eds. L.L. Sohn, L.P. Kouwenhoven, and G. Schön, NATO ASI Series E, vol. 345, pp. 105-214 (Kluwer, Dordrecht, 1997).

[3] A.C. Hewson, The Kondo Problem to Heavy Fermions, Cambridge University Press, Cambridge (1993).

[4] D. Goldhaber-Gordon et al., Nature 391, 156 (1998); S.M. Cronenwett et al., Science 281, 540 (1998); F. Simmel et al., Phys. Rev. Lett. 83, 804 (1999); J. Schmid et al., Phys. Rev. Lett. 84, 5824 (2000); W. G. van der Wiel et al., Science 289, 2105 (2000).

[5] L.I. Glazman and M.E. Raikh, Sov. Phys. JETP Lett. 47, 452 (1988); T.K. Ng, P.A. Lee, Phys. Rev. Lett. 61, 1768 (1988).

[6] J. Paaske, A. Rosch, J. Kroha, P. Wölfle, Phys. Rev. B 70, 155301 (2004).

[7] J. Elzerman et al., J. Low Temp. Phys. 118,375 (2000).

[8] A. Kogan, S. Amasha, and M.A. Kastner, Science 304, 1293 (2004).

[9] Q.-F. Sun and H. Guo, Phys. Rev. B 64, 153306 (2001).

[10] E. Lebanon and A. Schiller, Phys. Rev. B 65, 035308 (2002).

[11] R. Deblock, E. Onac, L. Gurevich, and L. P. Kouwenhoven, Science 301, 203 (2003).

[12] P. Nordlander, M. Pustilnik, Y. Meir, N. S. Wingreen, and D. C. Langreth, Phys. Rev. Lett. 83, 808 (1999).

[13] K. G. Wilson, Rev. Mod. Phys. 47, 773 (1975); T.A. Costi, A.C. Hewson, and V. Zlatić, J. Phys.: Cond. Mat. 6, 2519 (1994); W. Hofstetter, Phys. Rev. Lett. 85, 1508 (2000).

[14] W. Izumida, O. Sakai, and Y. Shimizu, J. Phys. Soc. Jpn. 66, 717 (1997).

[15] R. López, R. Aguado, G. Platero, and C. Tejedor, Phys. Rev. B 64, 075319 (2001).

[16] A.-P. Jauho, N.S. Wingreen and Y. Meir, Phys. Rev. B 50, 5528 (1994).

[17] S. Doniach and M. Sunjić, J. Phys. C: Solid State Phys. 3, 285 (1970). As shown by A. Rosch et al. [Phys. Rev. B 68, $014430(2003)]$ the $(1 / \sqrt{\omega})$-dependence is a very good heuristic description for $20 k T_{K}<\omega<200 k T_{K}$ before crossing over into a $\left(1 / \ln ^{2} \omega\right)$-dependence for larger frequencies (beyond the range of our numerical data).

[18] D.C. Langreth, Phys. Rev. 150, 516 (1966).

[19] A. Kaminski, Yu.V. Nazarov, and L.I. Glazman, Phys. Rev. B 62, 8154 (2000).

[20] $T_{K}$ corresponds to the frequency at which the spin spectral function takes its maximum. 